\title{
Agricultural research in the Global South: steering research beyond impact promises
}

\author{
Etienne HAINZELIN - Danielle BARRET - Guy FAURE \\ Marie-Hélène DABAT - Bernard TRIOMPHE
}

The impacts of agricultural research for development are long-term and very diverse - positive, unexpected, sometimes negative. To assess and understand these impacts, ImpresS, a participatory evaluation method that incorporates the viewpoints of actors on the ground, was tested on 13 research case studies conducted by CIRAD and its partners in different countries. The central conclusion is that research institutions and their funders need to change their practices if they wish to achieve long-term impacts at scale. For research, this means accepting to play multiple roles, collaborating with innovation and policy actors, fostering learning, and developing explicit hypothetical but plausible ex ante impact pathways. For sponsors and funders, it implies considering a wider range of impacts, planning action in the long term, fostering articulation between projects with similar goals, and supporting adaptive learning and management.

\section{Applied research is under increasing pressure to demonstrate its social utility}

Agriculture is at the heart of many burning global issues, and agricultural research for development (AR4D) is increasingly called upon to address multiple social challenges related to demographic, food system, ecological and climate transitions. However, funding for AR4D is shrinking. Funders and policy makers are asking researchers and their institutions to demonstrate convincingly not only how public investments in research generate excellent scientific results, but also how they contribute to producing innovations that have tangible impacts on development. Such impacts may relate, for instance, to food security, sustainable development or climate change adaptation.

This expectation is explicit in funding calls, and has led researchers to promise to deliver short-term impacts in their proposals. However, such promises often tend to be a rhetorical exercise, since impacts are seldom monitored over the long term. To respond to this issue, CIRAD has decided to invest in developing a "culture of impact" that aims to change research practices lastingly, increasing the ability of research to achieve development impacts.

\section{ImpresS: a participatory method}

\section{to assess research impacts}

In order to better understand its contribution to innovation processes in the Global South and to measure its impacts, CIRAD developed the ImpresS evaluation method (IMPact of RESearch in the South), which aims to answer the following questions ex post:

$>$ What are the lasting changes in society catalysed by research interventions?

$>$ How did these changes come about and why?

$>$ What was the actual contribution of research?

$>$ How diverse, intense and far-reaching are the impacts associated with these changes?

ImpresS adopts a participatory approach that incorporates the viewpoints of the different actors involved in a given innovation process. In 2015, the Impres $S$ method was applied to 13 case studies covering clusters of projects over the long 
term, illustrating the diversity of research conducted by CIRAD and its partners in a variety of contexts and partnership set-ups in Southern countries. These case studies span three continents and a broad range of technical and social innovations.

The ImpresS method starts by reconstructing the narrative of the innovation process and identifying its different phases. A timeline represents this narrative, while a map of key actors shows their interactions all along the innovation process. It then identifies the different positive or negative impacts produced by the innovation process, as reported by actors. Finally, based on the "impact pathway" concept, ImpresS identifies research outputs and describes how actors appropriate them, leading to changes in practices or organisations [outcomes] related to identified impacts.

ImpresS thus seeks to establish causal links by analysing the contribution of research at every stage of the impact pathway. This analysis emphasises capacity strengthening for the different stakeholders, identifying learning situations. Interactions with public policy actors are also of special interest. Key actors involved in the innovation process or affected by the changes it brings about contribute to the evaluation at different moments. Participatory tools make it possible to better understand the causal mechanisms and to identify diverse, sometimes unexpected impacts. The actors involved thus helped to identify more than 100 impacts for the 13 case studies. Each impact is characterised by quantitative and qualitative indicators emerging from the participatory process, and relates to one of 11 generic domains of impacts proposed by CIRAD. An expert panel grades the achievements of the case studies in each impact domain based on the impact indicators.

\section{Key lessons from ImpresS}

Interacting with all actors is critical to generate impacts • The 13 CIRAD case studies have shown the importance of researchers interacting with other actors at every stage of the innovation process. In the impact pathway, these interactions produce a series of outcomes representing essential steps towards impact: changes in farming or management practices, coordination mechanisms, innovation platforms, new organisations, the creation of companies, and the drafting of standards or policies. As with outcomes, many outputs are actually co-produced in the context of participatory research involving researchers and other actors. This co-production implies that the researchers operate within spheres that facilitate or structure such interactions. Researchers are also expected to interact with a range of political actors, who play a pivotal role in the construction, appropriation and especially the scaling of an innovation.

Capacity strengthening during the innovation process is a catalyst for impacts • In order to understand how capacity strengthening contributes to impacts, ImpresS identified the formal and informal learning situations that play a part in the construction of outputs and outcomes. Researchers participate directly or indirectly in these learning situations. The capacities thus built are diverse - technical, managerial, the ability to experiment, learn, or interact with others and their importance varies according to the innovation in question. They enable the actors concerned, including researchers, to enhance their capacity to innovate.

Impacts are diverse and built over the long term - The impacts identified and characterised for the 13 case studies are manifold: an increase in production and income, better natural resource management, improved access to remunerative markets, new public policies, etc. Some impacts differ from those anticipated by research and may even be negative. In most cases, impacts appeared only in the long term, after 20 years or more, and resulted from the successive contributions made by several projects rather than by individual projects.

Impact pathways are built over much longer periods than research projects. Moreover, the impacts observed in the long term are often not those initially planned. This unpredictable, long term process means it is neither possible nor advisable to plan research based on the promised impacts of a single project in isolation. Researchers' and funders' practices need to evolve; they need to operate on longer timescales and to reflect a better understanding of the mechanisms that generate impacts in order to boost their ability to achieve them.

\section{Range of impacts for varietal improvement of rainfed upland rice in Madagascar: ImpresS grading established by the expert panel, according to 11 impact domains.}

The length of bars represents impact intensity, from -5 to +5 , and the width of bars represents impact scope, from 1 to 3 .

The 11 axes of the radar represent the 11 impact domains identified by CIRAD; they correspond to five Sustainable Development Goals (SDGs): responsible consumption and production (SDC 12); no poverty and zero hunger (SDGs 1 and 2); partnerships for the goals (SDG 17); and life on land [SDG 15). In the example, five impact domains are concerned, corresponding to SDGs 1,2 and 12 .

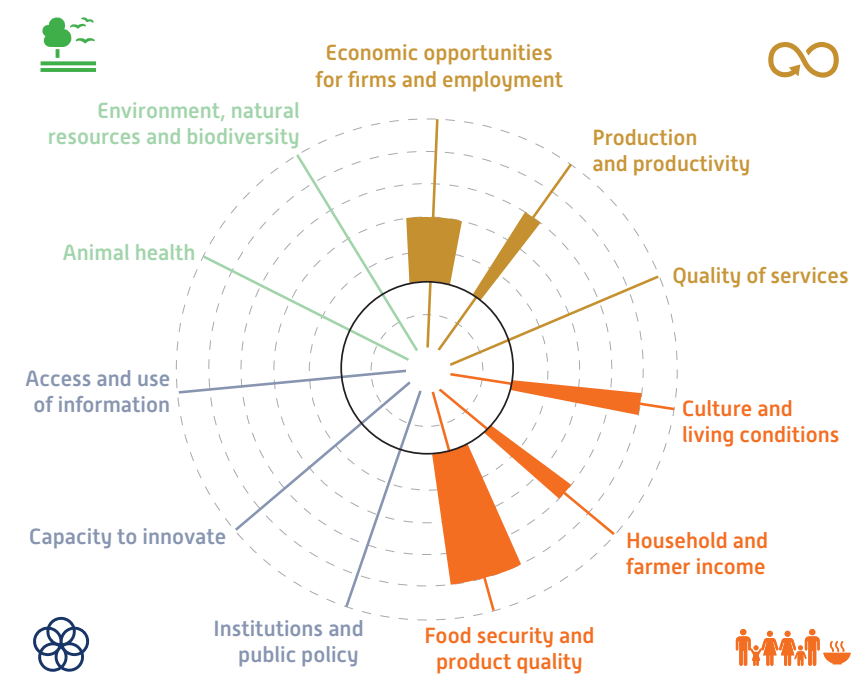




\section{Implications for research institutes}

Assuming the multiple roles of research and engaging in innovation systems - The specific context in which an innovation is developed means that research needs to assume multiple roles in order to generate impacts, in addition to its traditional knowledge production role. Based on the 13 CIRAD case studies, we have identified five generic roles played by research at different stages of the impact pathway: producing and disseminating knowledge; co-designing innovations; managing resources needed for actions; building capacity; and supporting actors and promoting the innovation. These roles are particularly diverse in the Global South because of relatively weaker public policies and development and advisory institutions.

For a given innovation process, the relative emphasis to be placed on these different research roles depends on the importance of scientific knowledge, on the willingness and capacity of actors to engage in the innovation process and, finally, on the overall socio-technical context. An individual researcher cannot play all of these roles, and they should instead be assumed collectively: research projects therefore need to pay greater attention to how to allocate tasks and orchestrate them over time.

Fostering interactions with innovation actors and political actors - To maximise the chances of achieving impacts in research projects, it is essential to foster interactions with the different actors all along the impact pathway through innovation systems, networks or platforms. These interactions often result in co-produced innovations, which are more relevant and more likely to produce impacts if they start occurring early in the process.

Through these interactions, researchers must also facilitate capacity strengthening for all stakeholders. Learning situations may be formal, such as organised workshops, or informal, such as the repeated interactions taking place within an experimentation process. They may be planned or may arise as the project progresses.

Finally, it is essential to foster interactions between researchers and policy makers at different scales, upon whom the achievement of impacts and scaling often depend. This requires flexibility from researchers on how to interact with such actors, including through informal relationships, and the capacity to take part in coalitions for favourable public policies.

Setting out the hypotheses for impact pathways - When designing research projects, it is first important to put them in context by relating them to previous interventions, be they public or private projects and programmes, and then to those that would currently interact with it, or might do so in the future.

The next step entails going beyond the conventional identification of project outputs to produce ex ante hypotheses about what they will become, through multiple interactions that should occur between actors to produce outcomes and contribute to achieving impacts. These hypotheses concern critical points along the impact pathway, the different roles research will have to play, the partnerships that need to be
The 13 case studies analysed by ImpresS, CIRAD's participatory method to assess the impacts of research.

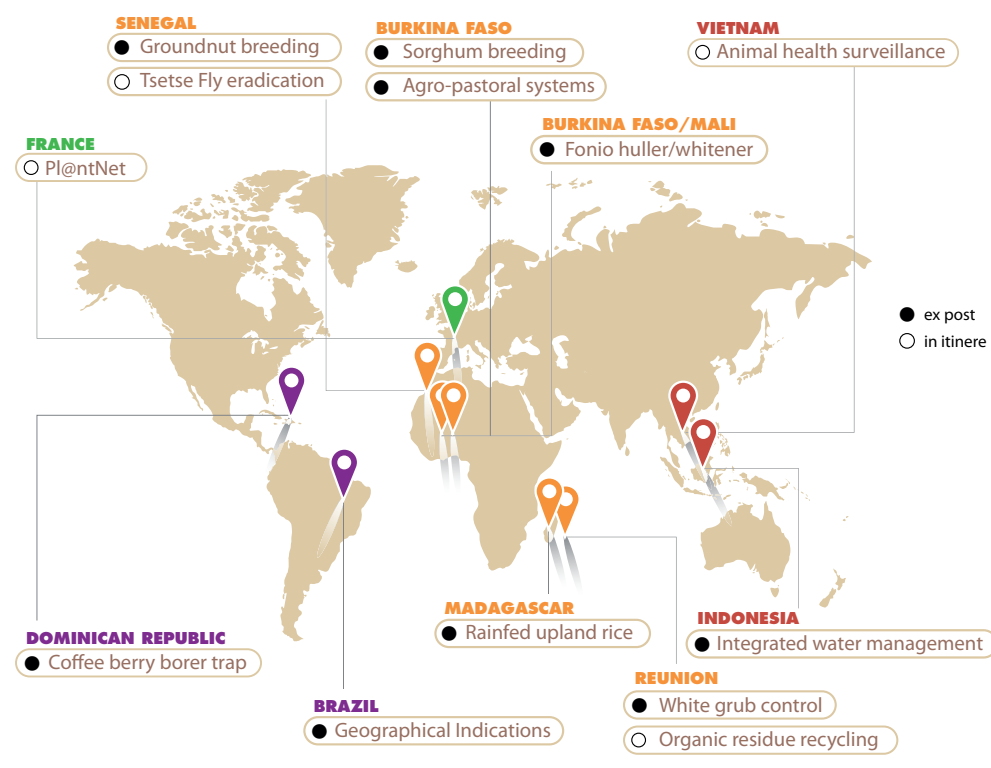

Illustration: Thierry Erwin (CIRAD, DSI)

in place, and more generally, the conditions for achieving these impacts. As far as possible, these hypotheses should be developed with the actors involved through a participatory approach. Lessons learned from previous experiences or projects may help to determine their plausibility.

\section{Implications for research sponsors and funders}

Considering a broad range of possible impacts - The fact that expected impacts, whether positive or negative, may emerge over long time spans has multiple implications. Beyond the strict agenda of funders or the research institution itself, and beyond specific demands that triggered research intervention, it is important to monitor all effects and impacts resulting from these actions. To do so, the 11 impact domains proposed by CIRAD, which relate to the Sustainable Development Goals, can be used as benchmarks. This also implies defining coherent clusters of projects in order to conduct an ex ante analysis of risks (potentially leading to negative impacts), and monitoring these over time.

Planning action in the long term - Because the economic, social, environmental and territorial impacts of research are produced over a long period (20 years or more), projects lasting 3 to 5 years are not an adequate or sufficient framework to obtain and measure these impacts. Taking on board the concept of a "cluster of projects" around a specific innovation trajectory, targeting a given region or population, would foster the continuity and capitalisation of research and partnerships with innovation actors. The ex ante impact hypotheses for research projects would then be based not only on a scientific literature review, but also on an analysis of previous or ongoing projects of a similar nature, with a view to better identifying their specific contribution to the desired impacts. 
Adjusting planning according to monitoring of impact hypotheses $\bullet$ In addition to ensuring sufficient involvement of different actors in the formulation of impact hypotheses during research design, projects need tools to monitor outcomes and impacts as they unfold through interaction with actors. Such monitoring enables eventual adjustments to project activities and planning. Funders should allow for a certain degree of flexibility and adaptive management by projects, which may imply changing their funding procedures in order achieve this.
Boosting the impact of research means letting go of the illusory promise of very short-term impacts and fostering among researchers and funders a better understanding of the underlying mechanisms that generate impacts over longer periods. Only a "culture of impact" that is based on this understanding, that capitalises on collective experience, uses rigorous tools and includes all actors involved in the innovation process, can guarantee the capacity to generate impacts.
Perspective $\mathrm{n}^{\circ} 42$ is based on CIRAD's Innovation-Impact Task force aimed at creating a methodological platform to assess the impact of research and to improve the culture of impact within the institution. This programme, conducted and financed by CIRAD, has resulted in the creation of the ex post impact assessment methodology known as ImpresS (IMPact of RESearch in the South] and its application to date to 13 innovation case studies involving research.

The ImpresS methodology and case studies are presented on the ImpresS website

http://impress-impact-recherche.cirad.fr/

as well as on CIRAD's website

www.cirad.fr/en/our-research/the-impact-of-our-research.

This programme has also given rise to the following publications:

Devaux-Spatarakis A., Barret D., Bouyer J., Cerdan C., Dabat M.-H., Faure G., Ferré T., Hainzelin E., Medah I., Temple L., Triomphe B., 2016. How can international agricultural research better contribute to innovations? Lessons from Impact pathways analysis. Communication in Social and technological transformation of farming systems: Diverging and converging pathways, European IFSA Symposium, Newport, 12-15 July 2016, 14 p. http://agritrop.cirad.fr/582679/.

Temple L., Biénabe E., Barret D., Saint-Martin G., 2016. Methods for assessing the impact of research on innovation and development in the agriculture and food sectors. African Journal of Science, Technology, Innovation and Development 8 [5-6]: 399-410. DOI: 10.1080/20421338.2016.1219484.

Triomphe B., Barret D., Clavel D., Dabat M.-H., DevauxSpatarakis A., Faure G., Hainzelin E., Mathé S., Temple L., Toillier A., 2015. Towards a generic, comprehensive and participatory approach for assessing the impact of agricultural research in developing countries. ImpAR Conference 2015: Impacts of agricultural research-towards an approach of societal values, INRA, Paris, 3-4 November 2015, 27 p. https://colloque.inra.fr/impar/Program-Material.

\section{A few words about...}

Étienne HAINZELIN is an agronomist. He is advisor to the President of CIRAD and a visiting professor at the University of Ottawa, Canada. He is cocoordinator of CIRAD's ImpresS initiative.

etienne.hainzelin@cirad.fr

Danielle BARRET is a specialist in management, policy and research assessment at CIRAD in the office of the director general. She is co-coordinator of the ImpresS initiative. danielle.barret@cirad.fr

Guy FAURE is director of INNOVATION Joint Research Unit at CIRAD (Innovation and development in agriculture and food, https://umr-innovation.cirad.fr/en). His research in management science focuses on advisory services for family farms and innovation processes in rural areas, especially the role of organisations in innovation. guy.faure@cirad.fr

Marie-Hélène DABAT is an economist at CIRAD in the ART-Dev Joint Research Unit (Actors, resources and territories in development http://art-dev.cnrs.fr/], specialising in assessment methodologies that she applies to projects, agricultural sectors and public policies. She is currently coordinating the VCA4D (Value Chain Analysis for Development) project for Agrinatura (http://agrinatura-eu.eu/).

marie-helene.dabat@cirad.fr

(2) This article is provided cc)(-) (2) under the terms of the BY NC SA Creative Commons License CC-BYNC-SA 4.0: Attribution-NonCommercialShare-Alike 4.0 International (https://creativecommons.org/licenses/by-nc-sa/4.0/deed.en).
To cite this document

Hainzelin E., Barret D., Faure G., Dabat M.-H. Triomphe B., 2017. Agricultural research in the Clobal South steering research beyond impact promises. CIRAD, Montpellier, Perspective 42 . DOI: $10.18167 /$ agritrop/00044
Bernard TRIOMPHE is a system agronomist at CIRAD in the INNOVATION Joint Research Unit. Currently based at IICA in Mexico (Inter-American Institute for Cooperation on Agriculture, http://www.iica.int/en), he has worked for many years on the participatory development of innovations and action research, with a focus on how multiple stakeholders, and especially local actors, contribute to innovation. bernard.triomphe@cirad.fr

THE IMPRESS INITIATIVE is supported by a methodological working group the coordinators of the case studies by CIRAD or its partners in the Global South, and involved a number of Masters students. We would like to take this opportunity to thank them all. 